\title{
Genetic consequences of human management in an introduced island population of red deer (Cervus elaphus)
}

\author{
DH Nussey ${ }^{1,2}$, J Pemberton ${ }^{1}$, A Donald ${ }^{2}$ and LEB Kruuk ${ }^{1}$ \\ ${ }^{1}$ Institute of Evolutionary Biology, University of Edinburgh, Edinburgh EH9 3JT, UK; ${ }^{2}$ Large Animal Research Group, Department \\ of Zoology, University of Cambridge, Downing Street, Cambridge CB2 3EJ, UK
}

\begin{abstract}
We investigated phylogeography and spatial genetic structure in an introduced island population of red deer (Cervus elaphus) on the Isle of Rum, Scotland, experiencing spatial variation in management regime. Five different mitochondrial DNA (mtDNA) haplotypes were present among female red deer on Rum. These comprised two phylogenetically divergent groups, one of which clustered with red deer from Sardinia and North Africa, while the other four grouped with other Western European red deer. Recent and historical red deer management practices explain this result. The Rum population is descended from recent introductions from at least four different UK mainland populations, and translocation of red deer within the UK and across Europe is well documented. We found significant spatial genetic structure across Rum in both mtDNA haplotypes and microsatellite markers. Mitochondrial spatial structure was over an order of
\end{abstract}

magnitude greater than structure in nuclear markers. This extreme difference is explained by the fact that the Rum population was introduced from different source populations, the highly male-biased dispersal patterns of red deer and the much smaller effective population size of mitochondrial compared to nuclear markers. Spatial structure in mtDNA conformed to a pattern of isolation by distance, while nuclear DNA did not. Apparent structure in the nuclear markers was driven by differences between the North Block and the rest of the island. We suggest that recent differences in the management regimes in different parts of the island have led to differences in effective male migration that would account for this observation.

Heredity (2006) 97, 56-65. doi:10.1038/sj.hdy.6800838; published online 17 May 2006

Keywords: management; phylogeography; spatial structure; dispersal; red deer; mtDNA

\section{Introduction}

Anthropogenic activity directly and indirectly influences the behaviour and demography of free-living animal populations and, as a result, may have a variety of consequences for spatial genetic structure (Harris et al, 2002). To date, the effects of human management practices on the population genetics of vertebrate game species have received little attention, despite their potential economic and conservation implications. The regular practice of human translocation of individuals between populations, often geographically distant from one another, in both domestic and game species can act to enhance genetic differentiation between populations, while also blurring a species' phylogeographic structure and undermining expected patterns of isolation by distance (Guiffra et al, 2000; Pereira et al, 2005). At the same time, selective culling to maximise some phenotypic quality (eg antler or horn size in males) is likely to alter levels of genetic variation or specific allele frequencies associated with these traits (Harris et al, 2002; Coltman et al, 2003a). Hunting may act to reduce spatial genetic structure if it is associated with disturbance to

Correspondence: DH Nussey, Large Animal Research Group, Department of Zoology, University of Cambridge, Downing Street, Cambridge CB2 3EJ, UK. E-mail: dhn24@cam.ac.uk

Received 1 November 2005; accepted 11 April 2006; published online 17 May 2006 social structure leading to increased migration, or generates spatial differences in mating opportunities that may encourage dispersal (Harris et al, 2002). Anthropogenic activity may also isolate such populations either through deliberate enclosure of managed stocks (eg fenced populations) or habitat fragmentation and human constructions preventing natural dispersal (Hartl et al, 1990), leading to increased genetic differentiation through genetic drift and mutation.

Red deer (Cervus elaphus) represent one of the largest extant game species in Europe, and many populations are subject to human management and trophy hunting (Whitehead, 1964; Long, 2003). There is little doubt that 'human intervention has drastically affected the natural genetic structure of red deer' (Gyllensten et al, 1983): the species has been the subject of regular human introductions from populations spread across the entire continent and beyond, since at least Roman times (Long, 2003). Indeed, records exist of introductions to UK populations from as far a field as North America with the aim of improving antler size (Long, 2003).

Analysis using mitochondrial DNA (mtDNA) has revealed genetic separation of $C$. elaphus into Eastern (including European, Middle Eastern and African subspecies) and Western (including East Asian and North American subspecies) clades or even species (Polziehn and Strobeck, 1998), with ancestral populations located in the Tarim region of southwest Asia (Ludt et al, 2004). 
Although seven geographic subspecies are currently recognised in Europe and Africa (Polziehn and Strobeck, 2002), genetic analysis has typically failed to support these taxonomic divisions (Gyllensten et al, 1983; Hartl et al, 1995). One notable exception is support from mitochondrial phylogenies for the taxonomic separation of red deer from the Tyrrhenian islands (Corsica and Sardinia) and North Africa from other mainland North and West European populations (Randi et al, 2001; Ludt et al, 2004).

Red deer exhibit a polygynous mating system, with male-biased dispersal. As is the case in many mammal systems, females are philopatric and remain close to their maternal relatives throughout their lives while males disperse from their natal area before reaching maturity (Clutton-Brock et al, 1982). Male-biased dispersal leads to the expectation of a greater than fourfold reduction in spatial structuring in nuclear genotypes relative to maternally inherited genetic material, such as mtDNA (Prugnolle and de Meeus, 2002). At finer spatial scales, female philopatry can result in clustering of related individuals and structuring of nuclear genotypes across continuous space (Coltman et al, 2003b; Nussey et al, 2005). Studies of red deer using nuclear DNA or protein markers have tended to find significant genetic structure between proximate populations of red deer in Europe, while failing to find evidence of any relationship between genetic and geographic distances between populations (Gyllensten et al, 1983; Hartl et al, 1990, 1995). This is just the pattern expected among populations subject to physical isolation and regular introductions from diverse source populations. However, few studies have been able to compare the effects of different management practices on population genetic structure across unfragmented or unfenced habitat in vertebrate game species (although see Hartl et al, 1991).
The population of red deer on the Isle of Rum, Scotland, represents a rare opportunity to explore the population genetic consequences of human translocation and differences in culling regimes. Existing records show deer were introduced to Rum from at least four different UK populations: Windsor Great Park, Knowsley Park in Lancashire, Meggernie Estate in Perthshire, and Warnham Park in Sussex (Marshall, 1998). As management of the island was handed over to government agencies in the 1950s, the red deer population on Rum has been subject to an annual 14\% cull (Clutton-Brock et al, 2002). During this period, the island has been separated into five 'management blocks', which are divided by physical structures such as valleys and mountain ridges, and a fenced area in the East around Kinloch village (see Figure 1). The physical features separating the management Blocks 1-5 (Figure 1) do not represent physical barriers to deer movement, indeed male dispersal between blocks often occurs (Clutton-Brock et al, 2002).

Since the late 1960s the red deer resident to the 'North Block' (Block 4; Figure 1) have been subject to long-term individual-based ecological, behavioural and genetic study (Clutton-Brock et al, 1982; Coulson et al, 2004; Nussey et al, 2005). Deer on the rest of the island have not been subject to individual-based study, but regular censuses of numbers have been taken (Clutton-Brock et al, 2002), and collection of bone and tissue samples from culls has also occurred. In this study, the management blocks are treated as subpopulations; since the 1970s each block has been treated to different management regimes. In 1973, the North Block of the island was completely released from culling, and consequently the female population density in this subpopulation increased while the number of males decreased (CluttonBrock et al, 2002). Between 1991 and 2000, Blocks 1 (south) and 3 (west) (Figure 1) were subject to increased
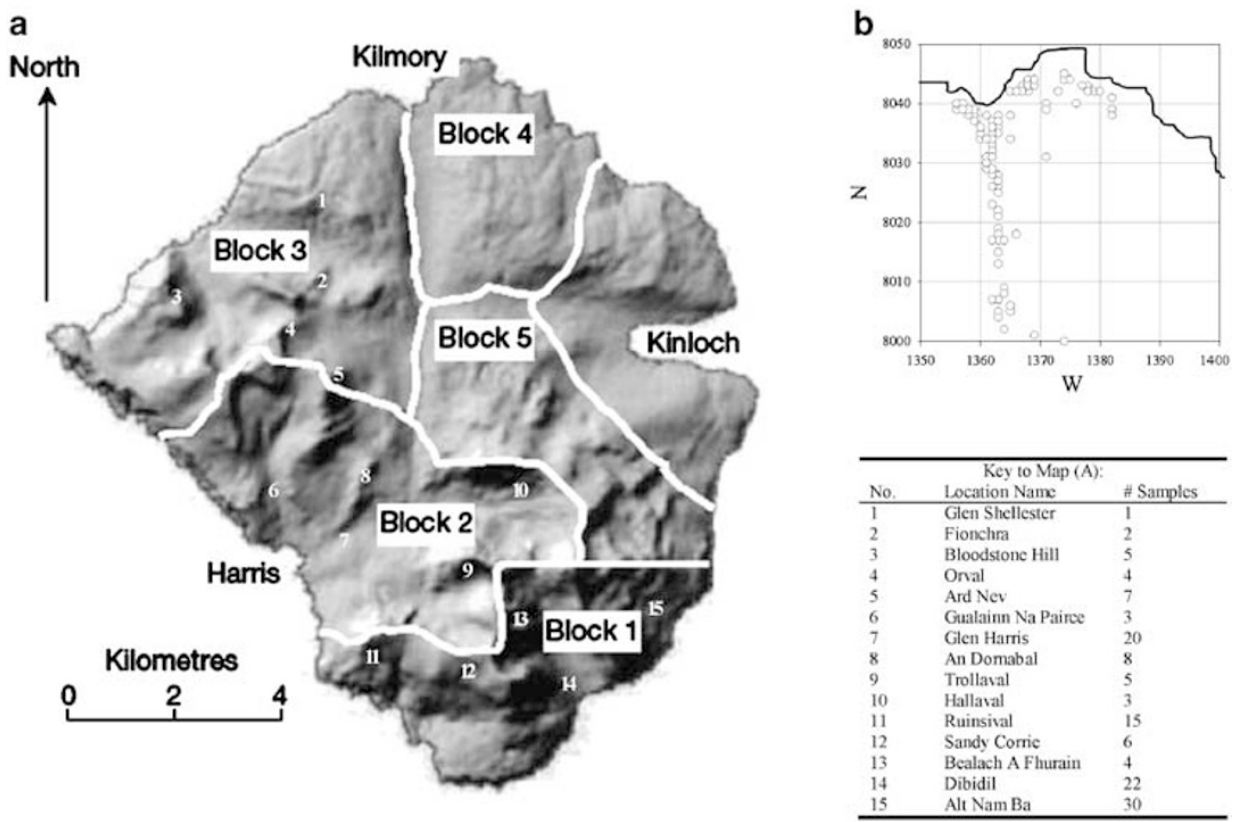

Figure 1 (a) Map of the Isle of Rum showing boundaries between four management blocks from which female red deer were sampled in this study. The numbers show locations of culls used in the analysis from Blocks 1 to 3; the location names and number of samples from each site are described in the key table. (b) Mean annual positions of female red deer aged 1 year or more and resident to Block 4 in 2001 . The $X$ - and $Y$ axis of this graph correspond to OS coordinates (north and west). 
male and female culls, respectively (Clutton-Brock et al, 2002). The increase in population density following the release from culling in Block 4 is known to have increased male emigration from this subpopulation, while the male cull in Block 1 seems to have increased male immigration into this area (Clutton-Brock et al, 2002).

The numerous introductions of red deer to Rum, coupled with the species' long history of translocation, could lead to the presence of phylogenetically differentiated mtDNA sequence variants within the island's population. Male dispersal and female philopatry would lead to strong spatial structuring of mtDNA variation. The degree of spatial structure in nuclear markers could reveal whether effective male dispersal across the island matches recently documented patterns of physical male migration (Clutton-Brock et al, 2002), and how this relates to recent management practices across Rum. Here, we use tissue samples from both the North Block study population and from culled females from the rest of the island to:

(i) Explore mitochondrial sequence divergence in the present day Rum population and relate this to existing molecular phylogeographic research into European red deer.

(ii) Compare spatial genetic structure of red deer across Rum in both mtDNA and nuclear markers to examine patterns of population genetic structure and sex-biased dispersal and to relate these to differences in recent management regimes across the island.

\section{Methods}

\section{Sample collection and selection}

Tissue samples for genotyping were collected from red deer from management Blocks 1-4 on the Isle of Rum (Figure 1; Clutton-Brock et al, 2002). A previous analysis has revealed evidence for spatial genetic structure among female red deer within the North Block, but no such structure among males (Nussey et al, 2005). With this in mind, we examined spatial genetic structure across Rum using genetic data from females in the present study.

Blocks 1-3: Tissue samples were collected from culled females on the Isle of Rum between October 2001 and January 2002. The location (to the nearest $1 \mathrm{~km}^{2}$ Ordinance Survey (OS) grid square) and age of the animals shot were obtained along with tissue samples. The 135 tissue samples from females aged 1 year or greater were obtained from a variety of localities across the island, from management Blocks 1-3. Figure 1 describes the approximate spatial distribution of the culls from which samples were obtained. The culling regime implemented in 2001 dictated the number of samples available from each Block: 77 samples from Block 1, 39 from Block 2 and 19 from Block 3 (Figure 1). Although, the sample sizes for the four subpopulations varied, the population genetic analyses that follow utilise estimates of population structure in which allele frequencies are weighted according to sample sizes within each subpopulation (Weir and Cockerham, 1984;
Goudet, 1995). Furthermore, dropping the block with the smallest sample size (Block 3) from these analyses produced the same patterns and estimates of very similar magnitude to the results presented below.

Block 4: Samples from Block 4 (the 'North Block' study area) females were collected as part of the ongoing research programme in this part of the island, rather than from culled individuals. Red deer the North Block have been subject to individual-based study since the early 1970s (Clutton-Brock et al, 1982). All individuals resident to the area are known from either artificial marks or natural idiosyncrasies (Clutton-Brock et al, 1982). Females in this population are matrilocal and rarely disperse beyond the Block's boundaries; female immigration into the population is also extremely rare (Coulson et al, 1997; Catchpole et al, 2004). The matriline of each deer born in Block 4 can be traced back to one female alive when the study began. Of 41 North Block matrilines with more than four female members, all matriarchs were born before 1975 and all but five of these matrilines can be traced back to females alive in the 1960s.

Since 1982 around $85 \%$ of calves born in Block 4 have been caught shortly after birth, and tissue samples taken. Tissue has also been taken from animals at immobilisation and post mortem. From these samples the majority of deer in the North Block born from 1980 onwards have been genotyped at up to 15 microsatellite loci (Marshall et al, 1998; Nussey et al, 2005). Detailed spatial data on the North Block animals has also been collected: since 1974 at least five censuses a month were undertaken between January and May, and the location (to the nearest $100 \mathrm{~m}^{2}$ OS grid square) of each individual seen on a census was noted. From this data residency of red deer to the North Block was defined (based on appearance in greater than $10 \%$ of January-May censuses; see Coulson et al, 1997) and the mean annual position of each resident deer was calculated (truncated to allocate deer to a $100 \mathrm{~m}^{2}$ OS grid square; see Nussey et al, 2005).

In all, 24 large to medium sized North Block matrilines ( $>15$ members of either sex throughout our study period), which had extant female members in 2001, were selected for mitochondrial analysis. From each, a maximum of four individuals' tissue samples were chosen for mitochondrial control region (mt CR) sequencing, avoiding maternal half-sib and mother-daughter pairs. In three matrilines samples from less than four individuals were available. A total of 91 tissue samples were selected for sequencing. Since mtDNA is maternally inherited and there was no evidence of different haplotypes within matrilines (see Results), we assumed identified mitochondrial haplotypes were shared within matrilines, and assigned mtDNA haplotypes to all individuals from selected matrilines accordingly.

In order to generate a contemporaneous and comparable genotypic sample to our cull samples from Blocks 1 to 3 , we identified 143 female red deer from the selected matrilines that were alive, aged 1 year or more, and resident to Block 4 in 2001 using census (January-May 2001) and life history data. Since the majority of natural mortality in this population occurs between January and April (Clutton-Brock et al, 1982), these animals were likely to have been those resident to the North Block when the 2001/2002 hind cull was occurring on the rest 
of the island. The sample of 143 haplotyped females from Block 4 was considerably larger than those available for the cull sampled blocks. To confirm this difference was not driving observed patterns, we re-ran our analyses of cross-island population structure ten more times, each time with a different random sample of 70 of these 143 females, so that the sample was of similar size to that of Block 1. This did not produce results of a different magnitude or pattern to those presented below.

\section{Genetic data}

Mitochondrial sequencing: A $922 \mathrm{bp}$ region of the mitochondrial control region ( $\mathrm{mt} C \mathrm{CR}$ ) was sequenced from 91 samples from Block 4 and 40 randomly selected cull samples from Blocks 1 to 3. DNA extraction was performed using either standard phenol-chloroform techniques or using Pharmacia ${ }^{\mathrm{TM}}$ cell and tissue extraction kits, or Qiagen ${ }^{\mathrm{TM}}$ DNAeasy tissue extraction kits. The mt CR was PCR-amplified using PRO (5'-CA CCATCAACCCCCAAAGCTGAAG- $\left.3^{\prime}\right)$ and PHE (5'CAGTGCCTTGCTTTGGGTTAAGC-3') primers (Wood and Phua, 1996). Each sample was amplified using one

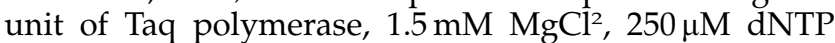
and $0.6 \mu \mathrm{M}$ of each primer. The following thermal cycle was used: $94^{\circ} \mathrm{C}$ for $2 \mathrm{~min}$, followed by 30 cycles consisting of $94^{\circ} \mathrm{C}$ for $30 \mathrm{~s}, 55^{\circ} \mathrm{C}$ for $30 \mathrm{~s}, 72^{\circ} \mathrm{C}$ for $1 \mathrm{~min}$, followed by $10 \mathrm{~min}$ at $72^{\circ} \mathrm{C}$. Successfully amplified PCR products were purified before sequencing using a SigmaGenosys GenElute $^{\mathrm{TM}}$ PCR clean-up kit. Sequencing was performed using DYEnamic ET Terminator Cycle Sequencing Kit (GE Healthcare Life Sciences, Munich, Germany), using three different primers in order obtain full sequence coverage of the selected $\mathrm{mt} C \mathrm{CR}$ region: PRO (5'-CACCATCAACCCCCAAAGCTGAAG-3'), HC3 (5'-CAGACGGCCATAGCTGAGTCCAAG-3') (Wood and Phua, 1996) and CST463 (5'-CTCGATGGACTAATGAC TAA-3') (Polziehn et al, 1998). Thermal cycling conditions for the sequencing reactions were as follows: 25 cycles consisting of $95^{\circ} \mathrm{C}$ for $20 \mathrm{~s}, 50^{\circ} \mathrm{C}$ for $15 \mathrm{~s}$ and $60^{\circ} \mathrm{C}$ for $1 \mathrm{~min}$. The fragments produced were analysed using either an ABI 377 or 3730 automated sequencer.

The sequences were aligned and consensus control region sequences produced for each individual using DNAStar $^{\mathrm{TM}}$ Sequence Manager. The 131 consensus sequences produced were aligned and compared using BioEdit (Hall, 1999). Two markedly different haplotype groups were evident from these initial alignments. One of these groups comprised a single invariant ('RUM A'). As the region of $\mathrm{mt} C \mathrm{CR}$ sequenced using the 'PRO' primer captures most of the nucleotide variation along this region in C. elaphus (Douzery and Randi, 1997; Randi et al, 2001) and the large sample of deer that was fully sequenced revealed no $\mathrm{mt} C \mathrm{CR}$ variation in RUM A haplotypes, we decided to use a combination of restriction enzyme digestion and $\mathrm{mt} C \mathrm{CR}$ sequencing using only 'PRO' to haplotype the remaining samples. Extraction and PCR of the remaining 95 samples from Blocks 1 to 3 were conducted as above. We then used the enzyme 'BstEII', which digested RUM A haplotype mt CR sequences at 350 base pairs from the $5^{\prime}$ end of the amplified region, but did not cut variants from the RUM $B$ haplotype group. Restriction digests were performed using $0.3 \mu \mathrm{l}$ of BstEII with $4 \mu \mathrm{l}$ of PCR product for $6 \mathrm{~h}$ at $60^{\circ} \mathrm{C}$. Digested PCR products of haplotype RUM A could be readily identified when run out on an agarose gel. Identified RUM B haplotype samples were then sequenced as above using only the PRO primer: the shorter sequence produced contained diagnostic sites for all four RUM B variants and no evidence of additional haplotypes were found in this sample.

Microsatellite genotyping: Microsatellite genotypes from the selected 143 North Block females at 10 polymorphic loci (FCB304, INRA011， INRA035，JP15， JP27，JP38, MAF109, RT1, TGLA94, TGLA322; see Supplementary Material for sequence and source data) were obtained from ongoing genotyping, undertaken for paternity analysis. The 135 DNA extractions from culled Block 1 to 3 females were genotyped at the same 10 loci (see Marshall et al, 1998 for further details of microsatellite genotyping techniques). Length polymorphisms were identified using an ABI 3730 automated sequencer and GeneMapper ${ }^{\mathrm{TM}}$ software.

\section{Data analysis}

Phylogenetic analysis of mitochondrial haplotypes: Phylogenetic and cladistic analyses were conducted in PHYLIP (Felsenstein, 1991) and TCS (Clement et al, 2000). All $\mathrm{mt} C R$ sequence variants observed were included in the analysis along with sequences covering the same region from European red deer available on GenBank (Table 1). UPGMA (based on Jukes-Cantor distances) and maximum parsimony trees were generated using the DNADIST, NEIGHBOR and DNAPARS modules in PHYLIP. The reliability of the branching patterns of these trees was assessed using 1000 bootstrap replicates of the sequence data. A haplotype network was also generated using statistical parsimony methods in TCS (Templeton et al, 1992).

Table 1 List of mitochondrial control region sequences from European and African red deer obtained from GenBank and used in phylogenetic analysis alongside Isle of Rum sequences presented here. The code for each sequence refers to those used in Figures 2 and 3

\begin{tabular}{lllll}
\hline Subspecies & Code & GenBank code & Location & Source reference \\
\hline C. e. atlanticus & ATLANT & AF291888 & Norway & Randi et al (2001) \\
C. e. hispanicus & HISPAN & AF291889 & Spain & Randi et al (2001) \\
C. e. corsicanus & CORSIC & AF291885 & Sardinia & Randi et al (2001) \\
C. e. hippelaphus & HIPPEL 86 & AF291886 & Southern Italy & Randi et al (2001) \\
C. e. hippelaphus & HIPPEL 87 & AF291887 & Southern Italy & Randi et al (2001) \\
C. e. barbarus & BARBAR & AF296808 & Algeria ${ }^{\mathrm{a}}$ & Polziehn and Strobeck (2002)
\end{tabular}

${ }^{a}$ The samples used for this sequence were actually from San Diego zoo and are described as the C. e. barbarus subspecies of red deer originating from Algeria in the source reference. 
Analysis of population structure: Population genetic structuring of both mtDNA and microsatellite markers was assessed by treating management blocks as separate subpopulations. Before analysis of structure the number of microsatellite alleles per locus were counted, and tests for linkage disequilibrium and deviation from HardyWeinberg equilibrium were conducted in these markers using FSTAT (Goudet, 1995). Estimates of global and pairwise $F_{S T}$ between Blocks were generated using ARLEQUIN (Schneider et al, 2000: mtDNA data) and FSTAT (microsatellite data). Global $F_{S T}$ values significantly greater than zero indicate significant structuring of genetic variation between groups. There was evidence of deviation from random mating within blocks in two of the microsatellite loci (INRA011 and JP27). To test the significance of global $\mathrm{F}_{\mathrm{ST}}$ estimates without assuming random mating within subpopulations, permutation tests based on 1000 randomisations of genotypes among subpopulations were run (Goudet, 1995). A fourfold difference in $\mathrm{F}_{\mathrm{ST}}$ values is expected between maternally and biparentally transmitted markers, as a result of differences in effective population size (Prugnolle and de Meeus, 2002). However, structuring in a maternally transmitted marker greater than four times larger than that for nuclear markers is typically interpreted as indicating male-biased dispersal (Prugnolle and de Meeus, 2002).

We also investigated spatial genetic structure at finer spatial scales using data on the location of culls from Blocks 1 to 3 and mean annual positions in 2001 from Block 4 (Figure 1). Analysis of the relationship between genetic and geographic distances between individuals were then performed separately for nuclear and mitochondrial data using SPAGeDi (Hardy and Vekemans, 2002). Spatial and genetic data were used to generate Moran's I statistics (Hardy and Vekemans, 1999) at each of the following distance intervals between pairs of individuals: $<1.5,1.5-3,3-6,6-9$ and $>9 \mathrm{~km}$. The significance of Moran's I-ln(distance) relationships was assessed by permuting the spatial group locations of individuals among all spatial groups (Hardy and Vekemans, 2002).

Since we found evidence from the microsatellite data that observed patterns of association between genetic and geographic distance might be driven by Block 4 alone, we re-ran the above analysis excluding data from Block 4 to further examine isolation by distance over the rest of the island.

\section{Results}

\section{Phylogenetic analysis of mitochondrial haplotypes}

Five different $m t$ CR haplotypes were found among the female red deer sequenced. The haplotypes fell into two main groups. The first, a single haplotype, which diverged from the other Rum haplotypes by between 2.8 and $3.0 \%$ of the $911 \mathrm{bp}$ region examined (11 sites with indels were excluded), was termed 'RUM A' (GenBank Accession Code: DQ386106). The second group included four more similar haplotypes, differing from each other by $0.6-1.3 \%$, were termed 'RUM B1-B4' (DQ386107Q386110). The most divergent of the Rum haplotypes were RUM A and RUM B3 with 27 substitutions across the $\mathrm{mt} C \mathrm{C}$ region. The haplotype pairs B1-B2, B1-B4 and
B3-B4 all differed by only five nucleotide substitutions, the lowest divergence across the Rum haplotypes.

Phylogenetic analysis revealed three clusters among the Rum haplotypes and the sequences from European red deer obtained from GenBank (see Table 1): the RUM A haplotype grouped the Sardinian and North African deer, the two Italian sequences grouped together, and the four RUM B haplotypes clustered with the Norwegian and Spanish red deer sequences (Figures 2 and 3).

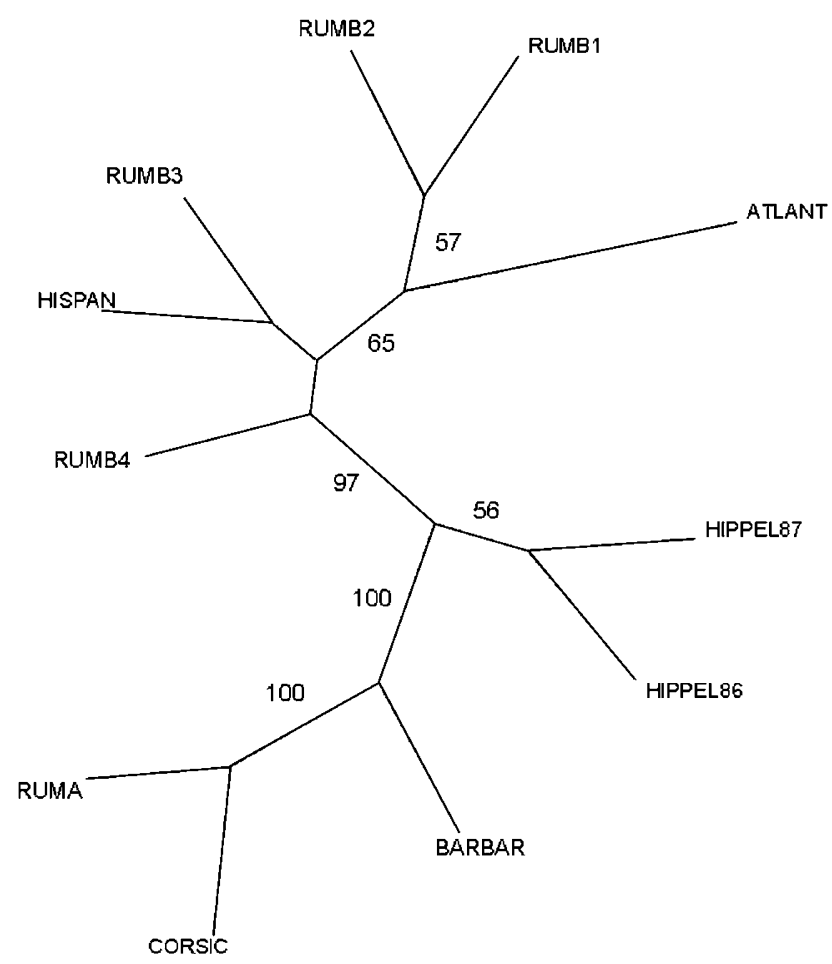

Figure 2 Unrooted maximum parsimony tree (unscaled) based on red deer mtDNA control region sequences observed on Rum ('RUM $\mathrm{A}^{\prime}$ and 'RUM B1' - 'RUM B4') and across Europe and North Africa (see Table 1 for descriptions of sequence labels). Analyses excluded sites featuring indels. Percentage consensus from 1000 bootstrap replications of the data are shown for branches where these values were greater than $50 \%$.

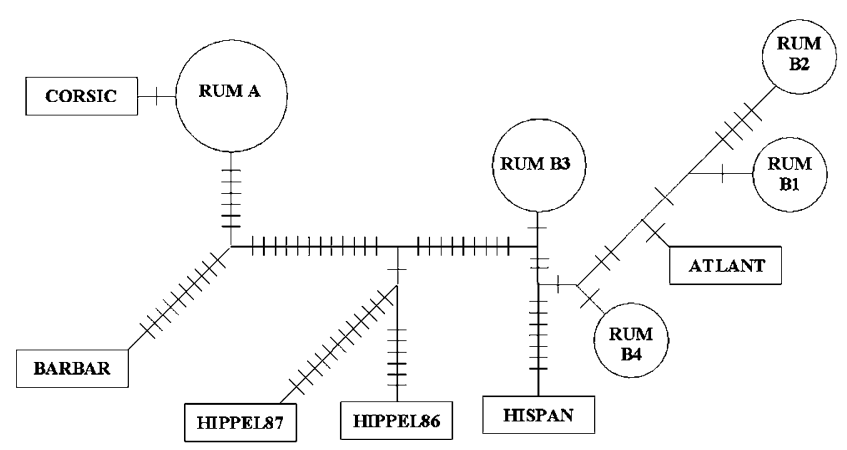

Figure 3 A parsimony network of red deer mtDNA control region sequences from Rum (circles) and across Europe and North Africa (rectangles; see Table 1 for descriptions of sequence labels). Sites including indels were treated as missing data. Each bar along a branch represents a single substitution. The size of the Rum haplotype circles roughly corresponds to the rank of their frequency across the island: RUM A was common (190), RUM B3 moderately common (57), and RUM B1, B2, and B4 were rare (16, 3, and 11, respectively). 
Maximum parsimony analysis generated four equally likely trees, all of which featured these three basic clusters and differed only in the branching patterns within the RUM B/Norway/Spain group (Figure 2). A consensus parsimony tree generated $100 \%$ bootstrap support for the RUM A/Sardinia/North Africa group, and 97\% support for the branch separating the RUM B/Norway/Spain cluster from the other haplotypes (Figure 2). The internal topography of this latter group was poorly resolved, and no branches were supported at over the $80 \%$ level by bootstrapping. The UPGMA tree (not shown) showed the same three main clades and a similar pattern of bootstrap support for branches. A parsimony network of these haplotypes (Figure 3), also supports the existence of three main groups, and illustrates the high levels of sequence divergence between these three groups of haplotypes. Figure 3 highlights the single substitution separating the RUM A and Tyrrhenian sequences, and shows the high levels of sequence divergence between these and the North African sequence.

\section{Analysis of population structure}

Mitochondrial DNA: The global $\mathrm{F}_{\mathrm{ST}}$ estimate for $\mathrm{mt} \mathrm{CR}$ haplotype frequencies was high and significantly greater than zero $\left(\mathrm{F}_{\mathrm{ST}}=0.373 ; P<0.001\right)$. Figure 4 shows the proportions of each mtDNA haplotype in each Rum management block. In Block 4, 21 of 24 sampled matrilines - 130 of $143(91 \%)$ resident females in 2001 were of the RUM A haplotype. $84 \%$ of culled females in Block 3 had RUM A haplotypes. In Block 2, 50\% of cull samples were RUM A, but haplotype RUM B3 was also reasonably common (31\%). These frequencies reversed in Block 1 with $59 \%$ of deer sampled being of RUM B3 haplotype and $32 \%$ of RUM A. Haplotype RUM B1 was found at low frequencies in Blocks 2, 3 and 4 (7-11\%), B2 was present only in a single matriline (three females alive in 2001) in Block 4, while B4 was only found in Blocks 1 and 2 (10\% in both blocks).

The spatial structure evident in mtDNA stems from the prevalence of the RUM A haplotype through the north and west of Rum (Blocks 3 and 4), whereas RUM B3 becomes increasingly common to the west and predominates in the south (Blocks 1 and 2; see Figure 4). A pattern of isolation-by-distance in highly philopatric female red deer was supported by pairwise $F_{\mathrm{ST}}$ estimates (Table 2) and spatial autocorrelation analysis (Figure 5a). Estimates in Table 2 revealed that neighbouring blocks had lower pairwise $\mathrm{F}_{\mathrm{ST}}$ values than non-neighbouring blocks (eg neighbouring blocks: $\mathrm{F}_{\mathrm{ST}}\left(4\right.$ vs 3) $=-0.005, \mathrm{~F}_{\mathrm{ST}}$ $(1$ vs 2$)=0.078$; non-neighbouring: $\mathrm{F}_{\mathrm{ST}}(4$ vs 2$)=0.323$,

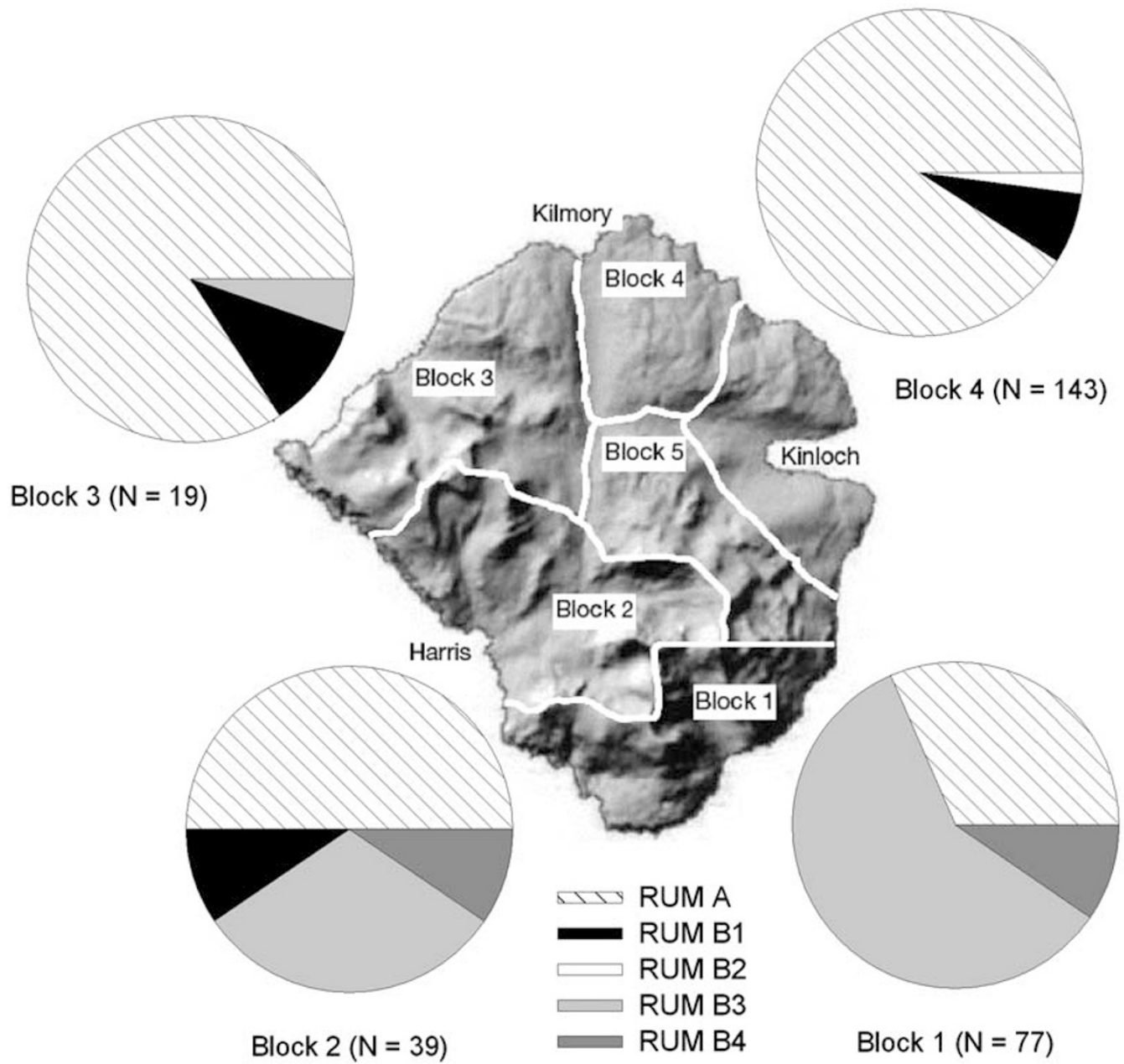

Figure 4 Map and pie charts showing the proportions of each mitochondrial haplotype found among female deer sampled in each of the management blocks; see key in figure for shading patterns representing different haplotypes. 

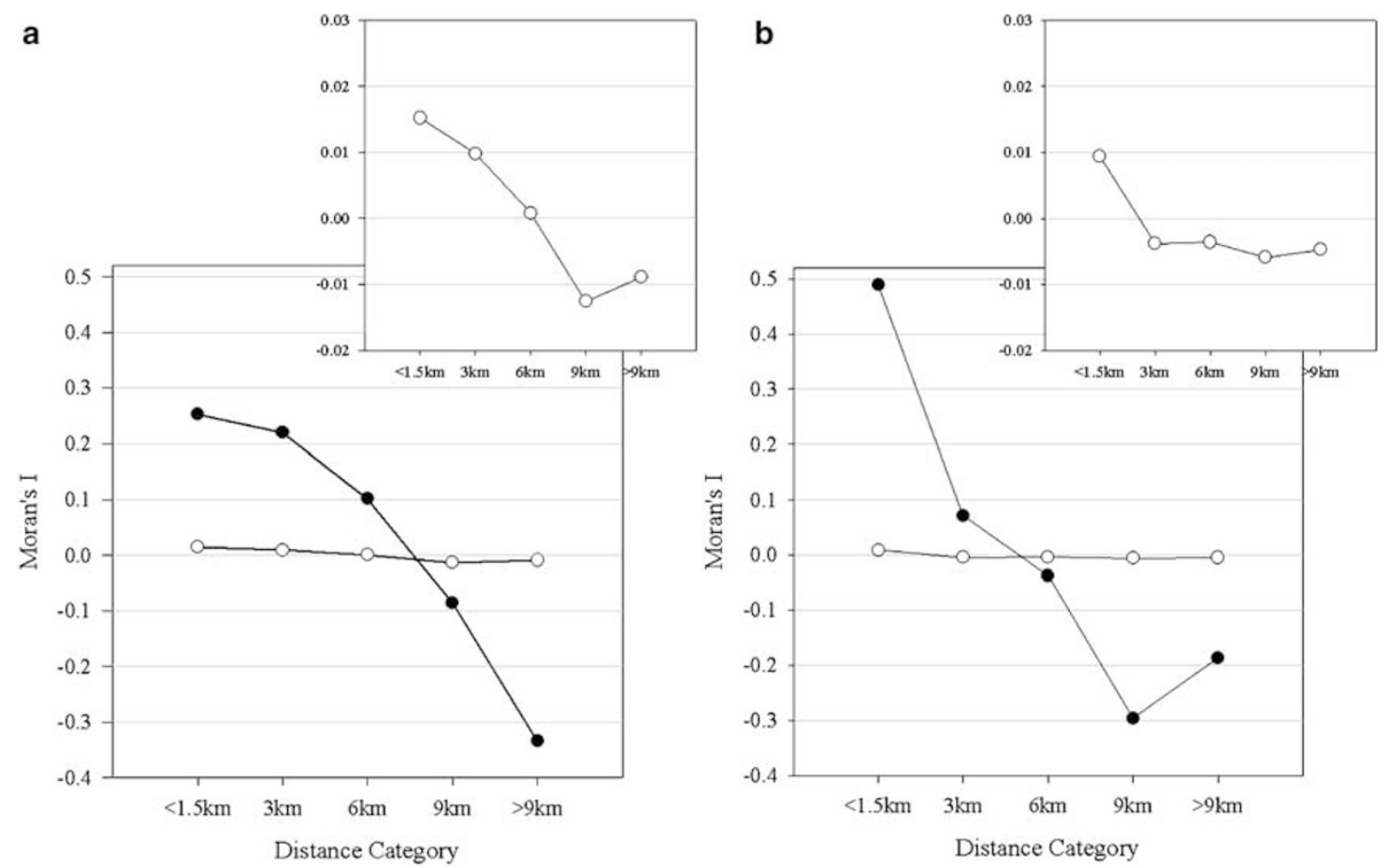

Figure 5 Correlograms, using Moran's I statistic as a measure of genetic spatial autocorrelation, for mtDNA and microsatellite data from female red deer on the Isle of Rum. (a) Correlogram based on all data for mtDNA (filled circles) and microsatellite markers (open circles, and inset with finer $Y$-axis scaling). (b) Correlogram based on same analysis as (a), except with females from Block 4 removed.

Table 2 Table showing pairwise $\mathrm{F}_{\mathrm{ST}}$ estimates between female red deer in culling Blocks 1-4 on Rum using mtDNA control haplotype frequencies (upper diagonal) and 10 polymorphic microsatellite loci (lower diagonal)

\begin{tabular}{lrccr}
\hline & 1 & 2 & 3 & \multicolumn{1}{c}{4} \\
\hline Block 1 & & $\mathbf{0 . 0 7 8}$ & $\mathbf{0 . 3 5 6}$ & $\mathbf{0 . 5 4 3}$ \\
Block 2 & -0.001 & & $\mathbf{0 . 1 2 2}$ & $\mathbf{0 . 3 2 3}$ \\
Block 3 & $\mathbf{0 . 0 0 7}$ & 0.005 & & -0.005 \\
Block 4 & $\mathbf{0 . 0 1 4}$ & $\mathbf{0 . 0 1 3}$ & $\mathbf{0 . 0 1 3}$ & \\
\hline
\end{tabular}

Estimates that were significantly greater than zero $(P<0.05)$ are indicated in bold.

$\mathrm{F}_{\mathrm{ST}}(3$ vs 1$\left.)=0.356\right)$. The correlogram for mtDNA in Figure 5a shows the probability of haplotype sharing decreases with the geographic distance between pairs of females: the estimated slope of Moran's I on ln(distance) was significantly negative $(b=-0.192, P<0.001)$.

Microsatellites: The number of alleles per microsatellite locus varied between six and 14 (see Supplementary Material), and there was no evidence for significant linkage disequilibrium between any loci. Two loci showed significant departures from Hardy-Weinberg equilibrium, INRA011 and JP27, but the permutation tests used to examine the significance of subpopulation differentiation did not assume random mating within blocks (see Methods). Global $\mathrm{F}_{\mathrm{ST}}$ for the microsatellites was more than an order of magnitude lower than that for the mitochondrial marker, although it was still significantly greater than zero $\left(\mathrm{F}_{\mathrm{ST}}=0.011 ; P<0.05\right)$. At finer spatial scales, the relationship between Moran's I and geographic distance was significantly negative
( $b=-0.011 ; P<0.001)$, but again more than an order of magnitude lower than the estimate for the mtDNA marker (Figure 5a).

Both microsatellite and mtDNA markers revealed higher pairwise estimates between Blocks 1 and $4(0.014$ and 0.543 , respectively) than between 1 and 3 (0.007 and 0.356), despite their being similar distances apart. This is explained by the presence of mountainous terrain (in Block 5, Figure 1a) and deer fencing presenting some obstacle to direct dispersal between Blocks 1 and 4 . More generally, pairwise $\mathrm{F}_{\mathrm{ST}}$ estimates for the microsatellite markers between pairs of blocks showed a different pattern to that observed for the mitochondrial marker (Table 2). There was no evidence of isolation by distance patterns and all values for comparisons involving Block 4 are greater than estimates involving other blocks (Table 2). The majority of structure observed across blocks in the nuclear markers may be driven by genetic differences between Block 4 and the rest of the island. To test this possibility we reanalysed both global $\mathrm{F}_{\mathrm{ST}}$ and isolation by distance patterns without Block 4 genotypes. There was still evidence of significant genetic spatial structure in $\mathrm{mtDNA}\left(\mathrm{F}_{\mathrm{ST}}=0.172, P<0.05 ; b=-0.207, P<0.01\right.$, Figure $5 b$ ), but no evidence of structure or isolation by distance in the microsatellite markers $\left(\mathrm{F}_{\mathrm{ST}}=0.003\right.$, $P>0.05 ; b=-0.0016 ; P>0.05$, Figure 5b).

\section{Discussion}

\section{Phylogeography of red deer on Rum: evidence of} translocation of females from across Europe and Africa The levels of mtDNA sequence divergence observed between red deer on the Isle of Rum would be 
startling if this were a natural, isolated island population. However, given that the population is descended from numerous introductions between the 1840s and 1920s from at least four mainland populations across the UK (Marshall, 1998), and that red deer have been the subject of extensive human translocation throughout the UK and Europe for many centuries (Whitehead, 1964; Hartl et al, 2003; Long, 2003), the high levels of divergence between mtDNA haplotypes on Rum are not surprising. Similar patterns of mitochondrial divergence within restricted geographic ranges have been observed in both this species (Feulner et al, 2004), and within breeds of domestic species (Guiffra et al, 2000; Pereira et al, 2005). Furthermore, there is good reason to expect similar levels of divergent matrilineal ancestry in other Scottish populations of red deer: records show regular introductions from populations in English parks across the mainland and islands throughout the last three centuries (Whitehead, 1964).

The phylogenetic analyses presented here suggest that red deer on Rum are descended from at least two geographically separate ancestral stocks (Figures 2 and 3). All three analyses conducted imply that the RUM A haplotype is very closely related to Tyrrhenian red deer, and also clusters with North African sequences. A recent phylogeographic study of red deer using the mtDNA cytochrome $b$ region, suggested that North African red deer colonised the Tyrrhenian islands and represent a taxonomic unit - an 'African' subspecies discrete from mainland European populations (Ludt et al, 2004; although see Zachos et al, 2003). The phylogeny presented here (Figure 2) provides further support for Ludt et al's (2004) suggestion of separate 'African' and 'Western European' taxonomic groups, utilising both a previously unanalysed combination of mt CR sequences (Table 1), and a different mtDNA region. The four RUM B haplotypes clustered with mainland European sequences and presumably are descended from several different West or North European stocks. It is impossible to be more specific until a more detailed phylogeographic analysis has been conducted, and even then extensive human translocation of this species may obfuscate any meaningful phylogeographic structure among European populations of red deer.

More detailed phylogeographic analyses of red deer in Europe and Africa are warranted to better understand their geographic origins and the extent of human translocation between regions. Furthermore, the presence of a close descendant of Tyrrhenian populations in a managed island population in the UK is potentially interesting from a conservation biology perspective. The Tyrrhenian subspecies, C. e. corsicanus, is currently listed as endangered by CITES (Jabbour et al, 1997) and there were thought to be less than 300 individuals remaining (Dolan, 1988 as cited by: Zachos et al, 2003). Although RUM A individuals have presumably undergone extensive introgression with Western European red deer, it may be possible to use historical records and sequence analysis to trace the source of this haplotype and identify extant populations of $C$. $e$. corsicanus descendants within UK populations which may have been less interbred with other red deer stocks.
Population structure and sex-biased dispersal: a consequence of different culling regimes?

The degree of genetic differentiation observed between culling blocks was almost 34 times higher in our mtDNA marker than in the microsatellite markers. The higher effective population size of nuclear DNA relative to maternally inherited DNA leads us to expect an approximately fourfold reduction in genetic structure from biparentally to uniparentally inherited genetic markers (Prugnolle and de Meeus, 2002). The differences in effective population size between nuclear and mtDNA markers, and the well-documented strong male-bias in dispersal in this species (Clutton-Brock et al, 1982, 2002) are sufficient to account for the observed inequality of magnitudes of the estimates of genetic structure between management blocks and at finer spatial scales between the markers types. The strong spatial structuring of mtDNA haplotypes may also result from nonrandom spatial patterns of introduction of deer to the island: different source populations, composed of different haplotypes may have been introduced to different parts of the island.

We found differences in the pattern of genetic isolation by distance between mtDNA and microsatellite markers. Pairwise $\mathrm{F}_{\mathrm{ST}}$ estimates suggested that individuals in Block 4 were responsible for most of the structure evident in the microsatellites, but that mtDNA showed a pattern expected under isolation by distance. Removal of Block 4 females from the data set backed this contention, suggesting that females from this area were responsible for much of the genetic structure in both global estimates of structure between blocks and finerscale patterns of spatial autocorrelation in the microsatellite data.

The observed demographic consequences of recent changes in management practices in the four blocks provide a plausible explanation for this observation. Following a release from culling in the North Block in 1973, the female population density in the block has increased to carrying capacity around which it has fluctuated since the mid-1980s (Clutton-Brock et al, 2002). The male population in the North Block concurrently declined, as a result of increased male emigration, decreased male immigration, and an increase in the male-bias of juvenile mortality (Clutton-Brock et al, 2002). The increasing population density and competition for food within Block 4 over the last three decades has resulted in declines in a variety of female and male fitness parameters (Clutton-Brock et al, 1987; Kruuk et al, 1999) and is likely to have resulted in a decrease in the phenotypic quality of males born in this region relative to the rest of the island. While levels of physical emigration of males from the North Block into other areas have certainly increased over this period (CluttonBrock et al, 2002; Catchpole et al, 2004), effective migration and hence gene flow from this block could have decreased if these dispersing males are in poor physiological condition relative to the males in neighbouring blocks, as they may be unable to successfully compete to hold harems and hence get access to oestrous females during the rut. Furthermore, rising resource competition or increasing difficulties maintaining harems when so many females are present in the North Block may discourage males from immigrating into the 
area during the rut to mate, although there are currently no data to support this contention. This possibility highlights the importance of genetic analysis in differentiating patterns of physical dispersal from effective dispersal of males in species showing skewed male reproductive success.

Changes to culling regimes across the rest of the island may also have contributed to the observed pattern in the microsatellite data. The experimentally increased cull of males in Block 1 since 1991 is thought to have attracted young males from neighbouring areas (Blocks 2 and 5) into the area, as overall male numbers in Block 1 proved hard to reduce (Clutton-Brock et al, 2002). This would have increased gene flow across the south of Rum young males from neighbouring blocks would presumably have excellent chances of mating with females in Block 1 given its reduced native male population - and is likely to have reduced any existing population genetic structure between Blocks 1 and 2 .

Human management practices are often hypothesised to reduce gene flow and hence lead to enhanced effects of genetic drift, and an eventual reduction in overall genetic variability. While it is not possible to exclude population processes that occurred before population monitoring began as an explanation for observed differences in spatial structuring of microsatellite genotypes across the island, recent changes in culling regimes on Rum represent a plausible explanation for the observed patterns of nuclear genetic structure among females. A recent study linked temporal changes in fine-scale population genetic structure among North Block females to changes in male and female effective population size, but not to changes in dispersal (Nussey et al, 2005). Across the Isle of Rum, differences in effective dispersal of males from and into Blocks 1 and 4 associated with recent changes in culling regimes appear to best explain patterns of female spatial genetic structure. This highlights potentially subtle interactions between human management strategies, environment, behaviour and phenotype affecting spatial genetic structure, which itself is a key factor determining the future evolutionary trajectory of any isolated population. Continued research examining cross-island population structure using older cull samples (eg before the experimental management changes began in 1991) or those collected from future culls are likely to further illuminate our understanding of how management practices and environmental quality affect dispersal behaviour and hence patterns of spatial genetic structure.

\section{Acknowledgements}

We thank Scottish Natural Heritage for permission to work on Rum, and their staff for support. We also thank Tim Clutton-Brock for managing the Kilmory red deer research project and Fiona Guinness, Sean Morris, and many other fieldworkers on the project. This work was funded by NERC (via a studentship to DHN and a Research Grant) and the Royal Society (LEBK). We are grateful to Ally Macaskill, John Alec Boyd and AnneMarie McMaster for collecting samples from the 2001 Rum hind cull. A great deal of help and hard work in the lab from Keith Allan, Kevin Connaghan, Sarah Lewis, Jon Slate and Kate Byrne were also involved in the work presented here. Finally, we thank Frank Zachos and two anonymous reviewers for their constructive comments on early drafts of this manuscript.

\section{References}

Catchpole EA, Fan Y, Morgan BJT, Clutton-Brock TH, Coulson T (2004). Sexual dimorphism, survival and dispersal in red deer. J Agr Biol Env Stat 9: 1-26.

Clement M, Posada D, Crandall KA (2000). TCS: a computer program to estimate gene genealogies. Mol Ecol 9: 1657-1659.

Clutton-Brock TH, Coulson TN, Milner-Gulland EJ, Thomson D, Armstrong HM (2002). Sex differences in emigration and mortality affect optimal management of deer populations. Nature 415: 633-637.

Clutton-Brock TH, Guinness FE, Albon SD (1982). Red Deer: Behaviour and Ecology of Two Sexes. University of Chicago Press: Chicago.

Clutton-Brock TH, Major M, Albon SD, Guinness FE (1987). Early development and population dynamics in red deer. I. Density-dependent effects on juvenile survival. J Anim Ecol 56: 53-67.

Coltman DW, O'Donoghue P, Jorgenson JT, Hogg JT, Strobeck C, Festa-Bianchet M (2003a). Undesirable evolutionary consequences of trophy hunting. Nature 426: 655-658.

Coltman DW, Pilkington JG, Pemberton JM (2003b). Fine-scale genetic structure in a free-living ungulate population. Mol Ecol 12: 733-742.

Coulson T, Guinness F, Pemberton J, Clutton-Brock T (2004). The demographic consequences of releasing a population of red deer from culling. Ecology 85: 411-422.

Coulson TN, Albon SD, Guinness FE, Pemberton JP, CluttonBrock TH (1997). Population sub-stucture, local density and calf winter survival in red deer (Cervus elaphus). Ecology 78 : 852-863.

Douzery E, Randi E (1997). The mitochondrial control region of Cervidae: evolutionary patterns and phylogenetic content. Mol Biol Evol 14: 1154-1166.

Felsenstein J (1991). PHYLIP: Phylogenetic Inference Package. 3.4. University of Washington: Seattle, WA.

Feulner PGD, Bielfeldt W, Zachos FE, Bradvarovic J, Eckert I, Hartl GB (2004). Mitochondrial DNA and microsatellite analyses of the genetic status of the presumed subspecies Cervus elaphus montanus (Carpathian red deer). Heredity 93: 299-306.

Goudet J (1995). Fstat version 1.2: a computer program to calculate F-statistics. J Heredity 86: 485-486.

Guiffra E, Kijas JMH, Amarger V, Carlborg O, Jeon J-T, Andersson L (2000). The origin of the domestic pig: independent domestication and subsequent introgression. Genetics 154: 1785-1791.

Gyllensten U, Ryman N, Reuterwall C, Dratch P (1983). Genetic differentiation in four European subspecies of red deer (Cervus elaphus L.). Heredity 51: 561-580.

Hall TA (1999). BioEdit: a user-friendly biological sequence alignment editor and analysis program for Windows 95/98/ NT. Nucleic Acids Symp Ser 41: 95-98.

Hardy OJ, Vekemans X (1999). Isolation by distance in a continuous population: reconciliation between spatial autocorrelation analysis and population genetics models. Heredity 83: $145-154$.

Hardy OJ, Vekemans X (2002). SPAGeDi: a versatile computer program to analyse spatial genetic structure at the individual or population levels. Mol Ecol Notes 2: 618-620.

Harris RB, Wall WA, Allendorf FW (2002). Genetic consequences of hunting: what do we know and what should we do? Wilson Soc Bull 30: 634-643.

Hartl GB, Lang G, Klein F, Willing R (1991). Relationships between allozymes, heterozygosity and morphological characters in Red Deer (Cervus elaphus), and the influence of selective hunting on allele frequency-distributions. Heredity 66: 343-350. 
Hartl GB, Nadlinger K, Apollonio M, Markov G, Klein F, Lang $G$ et al (1995). Extensive mitochondrial-DNA differentiation among European red deer (Cervus elaphus) Populations implications for conservation and management. Zeitschrift Fur Saugetierkunde-Int J Mammal Biol 60: 41-52.

Hartl GB, Willing R, Lang G, Klein F, Koller J (1990). Genetic variability and differentiation in red deer (Cervus elaphus L) of Central Europe. Genet Select Evol 22: 289-306.

Hartl GB, Zachos F, Nadlinger K (2003). Genetic diversity in European red deer (Cervus elaphus L.): anthropogenic influences on natural populations. Compt Rendus Biol 326: S37-S42.

Jabbour HN, Hayssen V, Bruford MW (1997). Conservation of deer: contributions from molecular biology, evolutionary ecology, and reproductive physiology. J Zool 243: 461-484.

Kruuk LEB, Clutton-Brock TH, Rose KE, Guinness FE (1999). Early determinants of lifetime reproductive success differ between the sexes in red deer. Proc Roy Soc London Ser B 266: $1655-1661$.

Long JD (2003). Introduced Mammals of the World: Their History, Distribution and Influence. CABI Publishing: Oxon.

Ludt CJ, Schroeder W, Rottmann O, Kuehn R (2004). Mitochondrial DNA phylogeography of red deer (Cervus elaphus). Mol Phylogenet Evol 31: 1064-1083.

Marshall TC (1998). Inbreeding and Fitness in Wild Ungulates. PhD, University of Edinburgh.

Marshall TC, Slate J, Kruuk LEB, Pemberton JM (1998). Statistical confidence for likelihood-based paternity inference in natural populations. Mol Ecol 7: 639-655.

Nussey DH, Coltman DW, Coulson T, Kruuk LEB, Donald A, Morris SJ et al (2005). Rapidly declining fine-scale spatial structure in female red deer. Mol Ecol 14: 3395-3405.

Pereira F, Pereira L, Van Asch B, Bradley DG, Amorim A (2005). The mtDNA catalogue of all Portuguese autochthonous goat (Capra hircus) breeds: high diversity of female lineages at the western fringe of European distribution. Mol Ecol 14: 2313-2318.

Polziehn RO, Hamr J, Mallory FF, Strobeck C (1998). Phylogenetic status of North American Wapiti (Cervus elaphus) subspecies. Can J Zool 76: 998-1010.

Polziehn RO, Strobeck C (1998). Phylogeny of Wapiti, Red Deer, Sika Deer, and other North American Cervids as determined from mitchondrial DNA. Mol Phylogenet Evol 10: 249-258.

Polziehn RO, Strobeck C (2002). A phylogenetic comparison of red deer and wapiti using mitochondrial DNA. Mol Phylogenet Evol 22: 342-356.

Prugnolle F, de Meeus T (2002). Inferring sex-biased dispersal from population genetic tools: a review. Heredity 88: 161-165.

Randi E, Mucci N, Claro-Hergueta F, Bonnet A, Douzery EJP (2001). A mitochondrial DNA control region phylogeny of the Cervidae: Speciation in Cervus and implications for conservation. Anim Conservat 4: 1-11.

Schneider S, Roessli D, Excoffier L (2000). Arlequin ver 2.000: A Software for Population and Genetics Data Analysis. Genetics and Biometry Laboratory, University of Geneva, Switzerland.

Templeton AR, Crandall KA, Sing CF (1992). A cladisticanalysis of phenotypic associations with haplotypes inferred from restriction endonuclease mapping and DNA-sequence data. 3. Cladogram estimation. Genetics 132: 619-633.

Weir BS, Cockerham CC (1984). Estimating F-statistics for the analysis of population structure. Evolution 38: 1358-1370.

Whitehead GK (1964). The Deer of Great Britain and Ireland. Routledge \& Kegan Paul: London.

Wood NJ, Phua SH (1996). Variation in the control region sequence of the sheep mitochondrial genome. Anim Genet 27: 25-33.

Zachos F, Hartl GB, Apollonio M, Reutershan T (2003). On the phylogeographic origin of the Corsican red deer (Cervus elaphus corsicanus): evidence from microsatellites and mitochondrial DNA. Mammal Biol 68: 284-298.

Supplementary Information accompanies the paper on Heredity website (http://www.nature.com/hdy). 